\title{
Quasinormal modes of maximally charged black holes*
}

\author{
Hisashi Onozawał Takashi Mishima ${ }^{\ddagger}$, Takashi Okamura, \\ and \\ Hideki Ishihara \\ Department of Physics, \\ Tokyo Institute of Technology, \\ Oh-okayama, Meguro, Tokyo 152, Japan \\ $\ddagger$ Laboratories of Physics, College of Science and Technology, \\ Nihon University, Narashinodai, Funabashi, Chiba 274, Japan
}

December 31, 1995

\begin{abstract}
A new algorithm for computing the accurate values of quasinormal frequencies of extremal Reissner-Nordström black holes is presented. The numerically computed values are consistent with the values earlier obtained by Leaver and those obtained through the WKB method. Our results are more precise than other results known to date. We also find a curious fact that the resonant frequencies of gravitational waves with multi-pole index $l$ coincide with those of electromagnetic waves with multi-pole index $l-1$ in the extremal limit.
\end{abstract}

\section{Introduction}

The determination of the quasinormal frequencies of black holes has for several years been of interest and is related to experiments which aim at detecting gravitational waves from supernovae or coalescences of binary neutron stars, which are thought to eventually form a black hole. In the late stage of the process of black hole formation, a certain mode of the gravitational wave dominates the emission. This is called the quasinormal mode of a black hole.

*TIT/HEP-311/COSMO-61

†E-mail:onozawa@phys.titech.ac.jp 
The equations governing oscillations of black holes are derived by Regge and Wheeler [1], and Zerilli[2] for the non-rotating uncharged case, Zerilli[3] and Moncrief [4] for the non-rotating charged case and Teukolsky [5] for the rotating uncharged case. The resonant frequencies of the Schwarzschild black holes were first computed by Chandrasekhar and Detweiler[6], who treated it as a boundary value problem of the second order ordinary differential equation of Regge-Wheeler. Integration of the Regge-Wheeler equation for the quasinormal boundary condition is numerically unstable. To avoid the instability, Leaver I7 presents the numerically stable continued fraction method for Schwarzschild and Kerr black holes. Here, a quasinormal mode function corresponds to a minimal solution of the recurrence relations which are satisfied by the coefficients of a series expansion of a wave function. The minimal solution of the threeterm recurrence relation is obtained by the corresponding continued fraction [8]. The continued fraction method can give the values of frequencies with high numerical precision because it uses no approximation, as is the case in the semianalytic WKB method developed in other works 9, 10, 11, 12. Leaver 13 also generalizes the continued fraction method to calculate accurate values of the quasinormal frequencies of Reissner-Nordström black holes, though Kokkotas and Schutz 14 raised the question of the applicability of the continued fraction method to charged black holes.

We are indeed motivated by his paper 13. There is another interesting question, that is, whether a continued fraction method can be used for the maximally charged black holes not discussed in his paper. In the extremal limit, the wave equation has an irregular singular point at the horizon, which makes the series expansion of a solution there invalid, and thus a continued fraction method does not seem to work. In this paper we show that a continued fraction method is applicable even to the extremal black holes if we expand a solution about a suitable ordinary point. In section II, we derive an eigenvalue equation with continued fractions after expanding a wave function about an ordinary point to determine the quasinormal frequencies of the extremal Reissner-Nordström black hole. In section III, we summarize the numerical results and find an interesting coincidence between the resonant frequencies of gravitational waves with multi-pole index $l$ and those of electromagnetic waves with multi-pole index $l-1$ in the limit of maximal charge. Section IV is devoted to conclusions and discussion.

\section{Eigenvalue Equation for Quasinormal Modes}

Leaver obtained the recurrence relations of Schwarzschild, Kerr[7], and ReissnerNordström black holes 13 by expanding solutions about the event horizon and then solved the characteristic equations with a continued fraction to get these resonant frequencies accurately. However his method is not valid when a black hole is extremal. The wave equation has an irregular singular point at the 
horizon about which the expansion of the solution is not available because a radius of convergence for a series expansion about an irregular singular point generally vanishes. Therefore we have to expand the solution about a suitable ordinary point in the extremal black holes.

We start from the Zerilli-Moncrief equation for perturbations on ReissnerNordström backgrounds of mass $M$ and charge $Q$ written as

$$
\left[\frac{d^{2}}{d r_{*}^{2}}+\omega^{2}-V_{s}(r)\right] Z_{s}(r)=0
$$

where

$$
\begin{aligned}
\frac{d r}{d r_{*}} & =\frac{\Delta}{r^{2}}, \\
V_{s} & =\frac{\Delta}{r^{5}}\left[A r-q_{s}+\frac{p_{s}}{r}\right], \\
\Delta & =r^{2}-2 M r+Q^{2}, \\
q_{0} & =2, \\
q_{1} & =3 M-\sqrt{9 M^{2}+4 Q^{2}(A-2)}, \\
q_{2} & =3 M+\sqrt{9 M^{2}+4 Q^{2}(A-2)}, \\
p_{0} & =2, \\
p_{1} & =p_{2}=4, \\
A & =l(l+1),
\end{aligned}
$$

and $s=0,1,2$ are for a massless scalar field, an odd parity electromagnetic perturbation, and an odd parity gravitational perturbation, respectively. The new parameter $p_{s}$ is introduced here in order to deal with the case of the scalar field as well. Note that the definitions of $q_{1}$ and $q_{2}$ are exchanged with each other from the book of Chandrasekhar 15, though the most of other notations follow this book. The scaling of $t$ and $r$ in this paper is such that $c=G=M=1$. We also use $\rho=-i \omega$ as a frequency variable for convenience.

Now we follow Leaver's procedure of getting a recurrence relation which plays a central role in a continued fraction method. The tortoise coordinate in the extremal limit becomes

$$
r_{*}=r+\log (r-1)^{2}-\frac{1}{r-1},
$$

which makes a difference in the basic equations between extremal and nonextremal black holes. The Zerilli-Moncrief equation (1) is therefore rewritten in terms of $r$-coordinate as follows,

$$
\begin{aligned}
& r(r-1) \frac{d^{2} Z_{s}}{d r^{2}}+2 \frac{d Z_{s}}{d r} \\
& -\left[\frac{\rho^{2} r^{5}}{(r-1)^{3}}+\frac{A r}{r-1}-\frac{q_{s}}{r-1}+\frac{p_{s}}{r(r-1)}\right] Z_{s}=0 .
\end{aligned}
$$


This equation has irregular singular points at $r=1$ and $r=\infty$ as we mentioned in the previous section, which makes the series expansion about the horizon invalid because it is hopeless that the series expansion is convergent. Hence it is necessary to improve Leaver's treatment of obtaining the quasinormal modes of the extremal black holes.

The boundary conditions of a quasinormal wave function are given by

$$
\begin{aligned}
Z_{s} \sim e^{-\rho r_{*}} \quad \text { as } & r_{*} \rightarrow \infty, \\
Z_{s} \sim e^{\rho r_{*}} \quad \text { as } & r_{*} \rightarrow-\infty,
\end{aligned}
$$

which mean there only exists purely outgoing wave at the infinity and purely ingoing wave at the black hole horizon. These are also transformed to

$$
\begin{aligned}
Z_{s} \sim r^{-2 \rho} e^{-\rho r} & \text { as } \quad r \rightarrow \infty, \\
Z_{s} \sim e^{\rho r}(r-1)^{2 \rho} e^{-\rho \frac{1}{r-1}} & \text { as } \quad r \rightarrow 1,
\end{aligned}
$$

which determine a pre-factor of a quasinormal wave function in the following form

$$
Z_{s}=e^{\rho r} e^{-\rho \frac{1}{r-1}}(r-1)^{2 \rho} r^{-4 \rho} e^{-2 \rho(r-1)} \sum_{n=0}^{\infty} a_{n} u^{n},
$$

where $u$ is specified by a point where we expand a solution. We use a series expansion about an ordinary point, which has a finite radius of convergence at least up to the nearest singular point. Thus, we use $u=(r-2) / r$ instead of $u=(r-1) / r$ which is used in Ref. [13]. In our choice of $u$ the black hole horizon and infinity correspond to $u=-1,1$, respectively. The distances from the point $u=0$ to two singular points $u=-1,1$ are equal, which can make us possible to examine the boundary conditions at both sides simultaneously. It is clear that the quasinormal mode boundary conditions are satisfied if and only if $\Sigma a_{n} u^{n}$ converges at both boundaries, horizon and infinity. This is equivalent to the condition that both $\Sigma a_{n}$ and $\Sigma(-)^{n} a_{n}$ are finite, that is, both $\Sigma a_{2 n}$ and $\Sigma a_{2 n+1}$ are convergent simultaneously. To examine the convergence of each summation, we first obtain the recurrence relation of coefficients $a_{n}$ of the expansion (17), and then attempt to obtain the recurrence relation only for $b_{n}=a_{2 n+1}$ and that of $c_{n}=a_{2 n}$. The quasinormal mode boundary conditions are satisfied when the odd and even sequence are minimal solutions of the corresponding recurrence relations, respectively.

After substituting the above series expansion (17) into Eq.(12) we obtain a five-term recurrence relation in the following,

$$
\begin{aligned}
\alpha_{1} a_{2}+\gamma_{1} a_{0} & =0, \\
\alpha_{2} a_{3}+\gamma_{2} a_{1}+\delta_{2} a_{0} & =0, \\
\alpha_{n} a_{n+1}+\beta_{n} a_{n}+\gamma_{n} a_{n-1}+\delta_{n} a_{n-2}+\epsilon_{n} a_{n-3} & =0,(n \geq 3),
\end{aligned}
$$


where the recurrence coefficients are given by

$$
\begin{aligned}
\alpha_{n} & =n^{2}+n, \\
\beta_{n} & =0, \\
\gamma_{n} & =-4 A-2 n^{2}-p_{s}+2 q_{s}+n(2-24 \rho)+12 \rho-64 \rho^{2}, \\
\delta_{n} & =2\left(p_{s}-q_{s}\right), \\
\epsilon_{n} & =n^{2}-p_{s}-12 \rho+16 \rho^{2}+n(-3+8 \rho) .
\end{aligned}
$$

For the scalar field, we can find that $\beta_{n}=\delta_{n}=0$ in the above relation, which means an odd sequence and even sequence are completely decoupled with each other, because the Zerilli-Moncrief equation in terms of $u$ is symmetric under the transformation $u \rightarrow-u$. Therefore it has only symmetric or anti-symmetric solutions as quasinormal mode functions. This is the same as the case of a quantum mechanical system with a reflection symmetry, where a wave function of energy eigenstate must be either symmetric or anti-symmetric. This makes the calculation of quasinormal frequencies of the scalar field easy. Each sequence is characterized by the three-term recurrence relation (20) starting from the two-term relation $(18)$ or $(19)$. We can obtain two independent characteristic equations below similar to one for the quasinormal frequencies of Schwarzschild black holes,

$$
\begin{aligned}
& \frac{a_{2}}{a_{0}}=-\frac{\gamma_{1}}{\alpha_{1}}=\frac{-\epsilon_{2}}{\gamma_{2}-} \frac{\alpha_{2} \epsilon_{4}}{\gamma_{4}-} \frac{\alpha_{4} \epsilon_{6}}{\gamma_{6}-} \cdots, \\
& \frac{a_{3}}{a_{1}}=-\frac{\gamma_{2}}{\alpha_{2}}=\frac{-\epsilon_{3}}{\gamma_{3}-} \frac{\alpha_{3} \epsilon_{5}}{\gamma_{5}-} \frac{\alpha_{5} \epsilon_{7}}{\gamma_{7}-} \cdots .
\end{aligned}
$$

These two equations are for the modes of even-numbered and those of oddnumbered overtone, respectively.

For gravitational or electromagnetic quasinormal modes, we have to develop a little more complicated way because the presence of $\delta_{n}$-term makes odd and even sequences dependent on each other, though it seems that they are not much correlated for large $n$ due to the fact that the coefficient $\delta_{n}$ becomes relatively small as $n$ increases compared with other terms. We first need to eliminate the odd or even sequence to examine the convergence of the summation of each sequence. This procedure is presented in Appendix A. For $b_{n}$ a five-term recurrence relation below is obtained,

$$
\begin{aligned}
\hat{\alpha}_{1} b_{2}+\hat{\beta}_{1} b_{1}+\hat{\gamma}_{1} b_{0} & =0, \\
\hat{\alpha}_{2} b_{3}+\hat{\beta}_{2} b_{2}+\hat{\gamma}_{2} b_{1}+\hat{\delta}_{2} b_{0} & =0, \\
\hat{\alpha}_{n} b_{n+1}+\hat{\beta}_{n} b_{n}+\hat{\gamma}_{n} b_{n-1}+\hat{\delta}_{n} b_{n-2}+\hat{\epsilon}_{n} b_{n-3} & =0,(n \geq 3),
\end{aligned}
$$

and for $c_{n}$ we get a following relation,

$$
\begin{aligned}
\bar{\alpha}_{2} c_{3}+\bar{\beta}_{2} c_{2}+\bar{\gamma}_{2} c_{1}+\bar{\delta}_{2} c_{0} & =0 \\
\bar{\alpha}_{n} c_{n+1}+\bar{\beta}_{n} c_{n}+\bar{\gamma}_{n} c_{n-1}+\bar{\delta}_{n} c_{n-2}+\bar{\epsilon}_{n} c_{n-3} & =0,(n \geq 3),
\end{aligned}
$$


where each recurrence coefficient is also given in Appendix A.

As mentioned previously, the quasinormal mode boundary conditions are satisfied if and only if both sequences are minimal solutions of recurrence relations simultaneously. In order to use a continued fraction method, both five-term recurrence relations are transformed to three-term recurrence relations by successive Gaussian elimination steps which are exhibited in Appendix B. Then, the ratios of the first two terms of both sequences for minimal solutions are determined by the corresponding continued fractions in the following,

$$
\begin{aligned}
& \frac{a_{4}}{a_{2}}=\frac{c_{2}}{c_{1}}=\frac{-\bar{\gamma}_{2}^{\prime \prime}}{\bar{\beta}_{2}^{\prime \prime}-} \frac{\bar{\alpha}_{2}^{\prime \prime} \bar{\gamma}_{3}^{\prime \prime}}{\bar{\beta}_{3}^{\prime \prime}-} \frac{\bar{\alpha}_{3}^{\prime \prime} \bar{\gamma}_{4}^{\prime \prime}}{\bar{\beta}_{4}^{\prime \prime}-} \cdots, \\
& \frac{a_{3}}{a_{1}}=\frac{b_{1}}{b_{0}}=\frac{-\hat{\gamma}_{1}^{\prime \prime}}{\hat{\beta}_{1}^{\prime \prime}-} \frac{\hat{\alpha}_{1}^{\prime \prime} \hat{\gamma}_{2}^{\prime \prime}}{\hat{\beta}_{2}^{\prime \prime}-} \frac{\hat{\alpha}_{2}^{\prime \prime} \hat{\gamma}_{3}^{\prime \prime}}{\hat{\beta}_{3}^{\prime \prime}-} \cdots,
\end{aligned}
$$

where the quantities with double-prime are recurrence coefficients of transformed three-term recurrence relations in Appendix B.

In contrast to scalar field case, the even and odd sequences are coupled each other through the non-vanishing $\delta_{n}$ terms. Dividing Eq.(20) for $n=3$ by Eq. (20) for $n=5$, we get the relation between the ratio of the first two terms of odd sequence and that of even sequence as follows,

$$
\begin{aligned}
\frac{a_{3}}{a_{1}} & =\frac{\alpha_{5} \frac{a_{6}}{a_{4}}+\gamma_{5}+\epsilon_{5} \frac{a_{2}}{a_{4}}}{\alpha_{2} \frac{a_{4}}{a_{2}}+\gamma_{3}+\epsilon_{3} \frac{a_{0}}{a_{2}} \frac{a_{2}}{a_{2}}} \\
& =\frac{-\frac{\alpha_{5}}{\alpha_{2}^{\prime \prime}}\left(\bar{\beta}_{2}^{\prime \prime}+\frac{a_{2}}{a_{4}} \bar{\gamma}_{2}^{\prime \prime}\right)+\gamma_{5}+\epsilon_{5} \frac{a_{2}}{a_{4}}}{\alpha_{3} \frac{a_{4}}{a_{2}}+\gamma_{3}-\epsilon_{3} \frac{\alpha_{1}}{\gamma_{1}}} \frac{a_{4}}{a_{2}},
\end{aligned}
$$

where the second equality comes from Eq.(18) and the transformed three-term relation (81) for $n=2$. The quasinormal mode boundary conditions are satisfied when the continued fractions (33) and (34) satisfy Eq.(35). This is the characteristic equation for the quasinormal frequencies of the extremal ReissnerNordström black hole.

Eq. 35 can be shifted an arbitrary number of times, $n$, to yield an equation for the ratio of successive terms of higher $n$ :

$$
\frac{a_{n+3}}{a_{n+1}}=\frac{\alpha_{n+5} \frac{a_{n+6}}{a_{n+4}}+\gamma_{n+5}+\epsilon_{n+5} \frac{a_{n+2}}{a_{n+4}}}{\alpha_{n+2} \frac{a_{n+4}}{a_{n+2}}+\gamma_{n+3}+\epsilon_{n+3} \frac{a_{n}}{a_{n+2}}} \frac{a_{n+4}}{a_{n+2}} .
$$

Eq. (35) and Eq.(36) are completely equivalent since every solution to (35) is also a solution to (36) when we substitute a continued fraction into each ratio of $a_{i} / a_{i-2}$, and vice versa. Eq.(36) can be used as a check of results obtained from Eq.(35). 


\section{Numerical Results}

The eigenvalue equations (26), (27) and (35) derived in the previous chapter are solved using the HYBRD subroutine distributed in MINPACK libraries. The three least-damped modes of $(s, l)=(2,2),(2,3),(2,4),(1,1),(1,2),(1,3)$, $(0,0),(0,1),(0,2)$ for the extremal Reissner-Nordström black hole are listed in Table 1 . We compare our results with Leaver's results 13 of nearly maximally charged case (99.99\% charged), which should be divided by two because of the difference of our scaling from his. These modes appear as complex conjugate pairs in $\rho$ because the eigenvalue equations are characterized by only real coefficients. We also compare them with the third order WKB quasinormal frequencies of the extremal Reissner-Nordström black hole. The WKB quasinormal frequencies are obtained using the same formula of Kokkotas and Schutz 14], who calculated the frequencies of charged black holes but did not show those of maximally charged black holes in the paper.

Our results are in agreement with other results obtained by Leaver and those obtained through the WKB method within the accuracy of a few percent. The difference between WKB frequencies and ours is ascribed to the fact that the WKB method gives only the approximate values. Indeed, the tendency that the discrepancy grows with the mode number suggests the breakdown of the WKB approximation for higher $n$. The difference between Leaver's results and ours is from the numerical error caused by the breakdown of his series expansion at the limit of maximal charge. Thus we are sure that our quasinormal frequencies of the extremal Reissner-Nordström black hole are the most accurate. Our results and his are plotted in Fig.1.

Notice that the quasinormal frequencies of $(s, l)=(2,2)$ and those of $(1,1)$ approach each other with increasing $Q$ and coincide in the extremal limit. We show this curious coincidence in the limit of maximal charge explicitly in the Fig.2. The trajectories of the first four lowest modes of the third order WKB gravitational quasinormal frequencies of $l=2,3,4,5$ are plotted. These quasinormal frequencies coincide with the frequencies of electromagnetic wave of $l=1,2,3,4$ in the limit of maximal charge, respectively. The first order WKB

quasinormal frequencies are given by $\omega_{n}^{2}=V_{\text {peak }}-i(n+1 / 2) \sqrt{-2 V_{\text {peak }}^{\prime \prime}}$ using the Zerilli-Moncrief Potential $V$, where a subscript $n$ is a mode number. The differences of $V_{\text {peak }}$ and $V_{\text {peak }}^{\prime \prime}$ between $V_{1}$ for $l=1$ and $V_{2}$ for $l=2$ are calculated to $\Delta V_{\text {peak }} / V_{\text {peak }} \simeq 2.79565 \times 10^{-16}$ and $\Delta V_{\text {peak }}^{\prime \prime} / V_{\text {peak }}^{\prime \prime} \simeq 5.46819 \times 10^{-15}$, respectively. This explains the coincidence of a few least-damped quasinormal frequencies of these two modes. Even for higher overtone modes $n \sim 30$, however, we numerically find a remarkable coincidence in the quasinormal frequencies of gravitational waves of $l$ and those of electromagnetic waves of $l-1$ for a wide range of multi-pole indices, $2 \leq l \leq 10$, through our method.

These two potentials, $V_{1}$ with multi-pole index $l$ and $V_{2}$ with multi-pole index $l+1$, indeed give the same transmission and reflection amplitude for each 
$\sigma$, whose evidence is presented in Appendix C. It implies that the quasinormal frequencies for both potentials are identical.

\section{Conclusions and Discussion}

We improve the continued fraction method of computing quasinormal frequencies of charged black holes in order to determine the quasinormal frequencies in the extremal limit and solve our formulae of eigenvalue equations by using a root search program. Our results of quasinormal frequencies for the maximally charged black holes are consistent with any other previous results 13, 14 and are the most accurate of all.

Our procedure is summarized as follows. We expand the solution about the ordinary point and get the recurrence relation of the expansion coefficients. In our method we have to examine the boundary conditions at both boundaries. Then, we need to divide the sequence into an odd sequence and an even sequence to examine convergence of both series. The convergence of the series at both sides occurs if and only if each sequence is minimal. The ratio of successive terms of each sequence is given by a continued fraction. Consequently, the eigenvalue equation is constructed by substituting the continued fraction into the ratio of successive recurrence relations.

The applicability of our method is free from types of singularities of the equation, in contrast to Leaver's method which is not useful when the equation has two irregular singular points at both boundaries. Though we can use our method to determine quasinormal frequencies of other black holes such as the Schwarzschild, non-extremal Reissner-Nordström and Kerr black holes, we actually did not follow this procedure in these black holes. For these black holes, his method is easier to obtain the quasinormal frequencies because the recurrence relation and the eigenvalue equation are simpler than ours. In our method, it is generally difficult to give the explicit forms of the recurrence relations of both even and odd sequences. In that case we will have to eliminate the even or odd sequence by numerical elimination steps. For the extremal Reissner-Nordström black hole, the recurrence relation, Eq.(20), is not so complicated in that it has no $\beta_{n}$-term and furthermore $\delta_{n}$-term has no dependence on $n$, which makes our procedure rather easy to accomplish.

We believe our method can be also used for the extremal Kerr black hole which has an irregular singular point at the horizon in the equation for radial part. Some authors 16, 17, 18] obtain the series of infinite numbers of quasinormal frequencies of the extremal Kerr black hole, which accumulate onto the critical frequency. Leaver also finds a tendency of an accumulation of quasinormal frequencies when the Kerr parameter approaches the maximum value. It is interesting to investigate what will happen to the distribution of quasinormal frequencies in the limit of maximal rotation, where Leaver's method is not valid. We are in preparation for this problem through our method of computing 
quasinormal frequencies.

We accidentally find a curious coincidence in the quasinormal frequencies of gravitational perturbations with multi-pole index $l$ and those of electromagnetic perturbations with $l-1$. These two modes are completely decoupled but they have the same amplitudes of transmission and reflection. In that case only the difference is a phase shift of transmitted or reflected wave, that means the quasinormal frequencies for both modes are identical. The situation is very similar to the coincidence of the transmission and reflection amplitudes of odd parity perturbations with those of even parity perturbation 15. At present there is no easy way to understand the hitherto unobserved coincidence but it is interesting that it occurs only in the extremal limit, where the black hole may have an unknown symmetry.

\section{Acknowledgments}

We would like to thank to Prof. A. Hosoya for his continuous encouragement. The research is supported in part by the Scientific Research Fund of the Ministry of Education.

\section{Appendix A}

Here we summarize a procedure to obtain a recurrence relation for the odd sequence and that for the even sequence of $a_{n}$. First we write down the recurrence relation, Eqs.(18), (19) and (20), in the matrix expression and separate the odd sequence from the even sequence as follows,

$$
\begin{aligned}
& \left(\begin{array}{ccccc}
\gamma_{1} & \alpha_{1} & & & \\
\epsilon_{3} & \gamma_{3} & \alpha_{3} & & \\
& \epsilon_{5} & \gamma_{5} & \alpha_{5} & \\
& & \ddots & \ddots & \ddots
\end{array}\right)\left(\begin{array}{l}
a_{0} \\
a_{2} \\
a_{4} \\
\vdots
\end{array}\right)=-\left(\begin{array}{llll}
0 & & & \\
& \delta & & \\
& & \delta & \\
& & \ddots
\end{array}\right)\left(\begin{array}{l}
0 \\
a_{1} \\
a_{3} \\
\vdots
\end{array}\right), \\
& \left(\begin{array}{ccccc}
\gamma_{2} & \alpha_{2} & & & \\
\epsilon_{4} & \gamma_{4} & \alpha_{4} & & \\
& \epsilon_{6} & \gamma_{6} & \alpha_{6} & \\
& & \ddots & \ddots & \ddots
\end{array}\right)\left(\begin{array}{l}
a_{1} \\
a_{3} \\
a_{5} \\
\vdots
\end{array}\right)=-\left(\begin{array}{llll}
\delta & & & \\
& \delta & & \\
& & \delta & \\
& & \ddots
\end{array}\right)\left(\begin{array}{l}
a_{0} \\
a_{2} \\
a_{4} \\
\vdots
\end{array}\right),
\end{aligned}
$$

where the term $\delta_{n}$ is constant therefore we write it as $\delta$ here, which makes us able to accomplish the below procedure explicitly. If we substitute the right 
hand side of Eq.(38) into the left hand side of Eq.(37) like this,

$$
\begin{aligned}
& \left(\begin{array}{ccccc}
\gamma_{1} & \alpha_{1} & & & \\
\epsilon_{3} & \gamma_{3} & \alpha_{3} & & \\
& \epsilon_{5} & \gamma_{5} & \alpha_{5} & \\
& & \ddots & \ddots & \ddots
\end{array}\right)\left(\begin{array}{ccccc}
\gamma_{2} & \alpha_{2} & & & \\
\epsilon_{4} & \gamma_{4} & \alpha_{4} & & \\
& \epsilon_{6} & \gamma_{6} & \alpha_{6} & \\
& & \ddots & \ddots & \ddots
\end{array}\right)\left(\begin{array}{l}
a_{1} \\
a_{3} \\
a_{5} \\
\vdots
\end{array}\right) \\
& =\left(\begin{array}{cccc}
0 & & & \\
\delta^{2} & 0 & & \\
& \delta^{2} & 0 & \\
& & \ddots & \ddots
\end{array}\right)\left(\begin{array}{l}
a_{1} \\
a_{3} \\
a_{5} \\
\vdots
\end{array}\right),
\end{aligned}
$$

the even sequence is eliminated and then we can obtain a recurrence relation including only $b_{n}$ which was presented in Eq.(30) and the recurrence coefficients are explicitly given by

$$
\begin{aligned}
\hat{\alpha}_{n}= & -3 n+n^{2}+8 n^{3}+4 n^{4}, \\
\hat{\beta}_{n}= & 4 n\left(-n-2 A n-4 n^{3}+n q_{s}+9 \rho-24 n^{2} \rho-32 n \rho^{2}\right), \\
\hat{\gamma}_{n}= & 8+12 A+4 l^{2}(l+1)^{2}+24 n^{4}-6 q_{s}-4 A q_{s}+q_{s}^{2} \\
& -70 \rho-48 A \rho+24 q_{s} \rho+308 \rho^{2}+128 A \rho^{2}-64 q_{s} \rho^{2} \\
& -768 \rho^{3}+1024 \rho^{4}+n^{3}(-48+224 \rho) \\
& +n^{2}\left(46+16 A-8 q_{s}-336 \rho+864 \rho^{2}\right) \\
& +2 n\left(-11-8 A+4 q_{s}+126 \rho+48 A \rho-24 q_{s} \rho-432 \rho^{2}+768 \rho^{3}\right), \\
\hat{\delta}_{n}= & 8+4 A-16 n^{4}-\left(4-q_{s}\right)^{2}-2 q_{s}+32 n^{3}(2-5 \rho)+68 \rho \\
& +32 A \rho-16 q_{s} \rho-440 \rho^{2}-32 A \rho^{2}+16 q_{s} \rho^{2}+896 \rho^{3} \\
& -512 \rho^{4}+4 n^{2}\left(-21-2 A+q_{s}+120 \rho-144 \rho^{2}\right) \\
& +4 n\left(10+4 A-2 q_{s}-97 \rho-8 A \rho+4 q_{s} \rho+288 \rho^{2}-224 \rho^{3}\right), \\
\hat{\epsilon}_{n}= & -15 n+41 n^{2}-24 n^{3}+4 n^{4}-30 \rho+164 n \rho-144 n^{2} \rho+32 n^{3} \rho \\
& +164 \rho^{2}-288 n \rho^{2}+96 n^{2} \rho^{2}-192 \rho^{3}+128 n \rho^{3}+64 \rho^{4} .
\end{aligned}
$$

Similarly we get a recurrence relation for $c_{n}$ and the recurrence coefficients are given by

$$
\begin{aligned}
\bar{\alpha}_{n}= & 1-5 n^{2}+4 n^{4} \\
\bar{\beta}_{n}= & (-1+2 n)\left(2+2 A-8 n-4 A n+12 n^{2}-8 n^{3}\right. \\
& \left.-q_{s}+2 n q_{s}+6 \rho+48 n \rho-48 n^{2} \rho+32 \rho^{2}-64 n \rho^{2}\right), \\
\bar{\gamma}_{2}= & 41+24 A+4 A^{2}-12 q_{s}-4 A q_{s}+q_{s}^{2}+306 \rho+96 A \rho-48 q_{s} \rho \\
& +948 \rho^{2}+128 A \rho^{2}-64 q_{s} \rho^{2}+1536 \rho^{3}+1024 \rho^{4}, \\
\bar{\gamma}_{n}= & 38+24 A+4 A^{2}+24 n^{4}-12 q_{s}-4 A q_{s} \\
& +q_{s}^{2}-308 \rho-96 A \rho+48 q_{s} \rho+956 \rho^{2}+128 A \rho^{2}
\end{aligned}
$$




$$
\begin{aligned}
& -64 q_{s} \rho^{2}-1536 \rho^{3}+1024 \rho^{4}+32 n^{3}(-3+7 \rho) \\
& +2 n^{2}\left(77+8 A-4 q_{s}-336 \rho+432 \rho^{2}\right) \\
& +4 n\left(-29-8 A+4 q_{s}+189 \rho+24 A \rho-12 q_{s} \rho-432 \rho^{2}+384 \rho^{3}\right),(48) \\
\bar{\delta}_{2}= & -8+4 A+6 q_{s}-q_{s}^{2}+12 \rho-12 A \rho+6 q_{s} \rho-76 \rho^{2} \\
& -16 A \rho^{2}+8 q_{s} \rho^{2}-336 \rho^{3}-256 \rho^{4} \\
\bar{\delta}_{n}= & -42-6 A-16 n^{4}-\left(4-q_{s}\right)^{2}+3 q_{s}+32 n^{3}(3-5 \rho) \\
& +402 \rho+48 A \rho-24 q_{s} \rho-1160 \rho^{2}-32 A \rho^{2}+16 q_{s} \rho^{2} \\
& +1344 \rho^{3}-512 \rho^{4}+4 n^{2}\left(-51-2 A+q_{s}+180 \rho-144 \rho^{2}\right) \\
& +4 n\left(45+6 A-3 q_{s}-247 \rho-8 A \rho+4 q_{s} \rho+432 \rho^{2}-224 \rho^{3}\right) \\
\bar{\epsilon}_{n}= & 21-76 n+83 n^{2}-32 n^{3}+4 n^{4}-152 \rho+332 n \rho-192 n^{2} \rho \\
& +32 n^{3} \rho+332 \rho^{2}-384 n \rho^{2}+96 n^{2} \rho^{2}-256 \rho^{3}+128 n \rho^{3}+64 \rho^{4} .(51)
\end{aligned}
$$

\section{Appendix B}

We give the Gaussian elimination steps to transform the five-term recurrence relations into three-term recurrence relations. For $b_{n}$ the step defined by

$$
\begin{aligned}
\hat{\alpha}_{n}^{\prime} & =\hat{\alpha}_{n}, \\
\hat{\beta}_{n}^{\prime} & =\hat{\beta}_{n}, \\
\hat{\gamma}_{n}^{\prime} & =\hat{\gamma}_{n}, \\
\hat{\delta}_{n}^{\prime} & =\hat{\delta}_{n}, \quad \text { for } n=1,2, \\
\hat{\alpha}_{n}^{\prime} & =\hat{\alpha}_{n}, \\
\hat{\beta}_{n}^{\prime} & =\hat{\beta}_{n}-\hat{\alpha}_{n-1}^{\prime} \hat{\epsilon}_{n} / \hat{\delta}_{n-1}^{\prime}, \\
\hat{\gamma}_{n}^{\prime} & =\hat{\gamma}_{n}-\hat{\beta}_{n-1}^{\prime} \hat{\epsilon}_{n} / \hat{\delta}_{n-1}^{\prime}, \\
\hat{\delta}_{n}^{\prime} & =\hat{\delta}_{n}-\hat{\gamma}_{n-1}^{\prime} \hat{\epsilon}_{n} / \hat{\delta}_{n-1}^{\prime}, \quad \text { for } n \geq 3,
\end{aligned}
$$

transforms the five-term recurrence relation into a four-term recurrence relation and the second elimination step below,

$$
\begin{aligned}
& \hat{\alpha}_{1}^{\prime \prime}=\hat{\alpha}_{1}^{\prime}, \\
& \hat{\beta}_{1}^{\prime \prime}=\hat{\beta}_{1}^{\prime}, \\
& \hat{\gamma}_{1}^{\prime \prime}=\hat{\gamma}_{1}^{\prime}, \\
& \hat{\alpha}_{n}^{\prime \prime}=\hat{\alpha}_{n}^{\prime}, \\
& \hat{\beta}_{n}^{\prime \prime}=\hat{\beta}_{n}^{\prime}-\hat{\alpha}_{n-1}^{\prime \prime} \hat{\delta}_{n}^{\prime} / \hat{\gamma}_{n-1}^{\prime \prime}, \\
& \hat{\gamma}_{n}^{\prime \prime}=\hat{\gamma}_{n}^{\prime}-\hat{\beta}_{n-1}^{\prime \prime} \hat{\delta}_{n}^{\prime} / \hat{\gamma}_{n-1}^{\prime \prime}, \quad \text { for } n \geq 2,
\end{aligned}
$$

brings us a final three-term recurrence relation in the following,

$$
\hat{\alpha}_{n}^{\prime \prime} b_{n+1}+\hat{\beta}_{n}^{\prime \prime} b_{n}+\hat{\gamma}_{n}^{\prime \prime} b_{n-1}=0, \quad \text { for } n \geq 1 .
$$


For $c_{n}$ the successive elimination steps in the following,

$$
\begin{aligned}
\bar{\alpha}_{2}^{\prime} & =\bar{\alpha}_{2}, \\
\bar{\beta}_{2}^{\prime} & =\bar{\beta}_{2}, \\
\bar{\gamma}_{2}^{\prime} & =\bar{\gamma}_{2}, \\
\bar{\delta}_{2}^{\prime} & =\bar{\delta}_{2}, \\
\bar{\alpha}_{n}^{\prime} & =\bar{\alpha}_{n}, \\
\bar{\beta}_{n}^{\prime} & =\bar{\beta}_{n}-\bar{\alpha}_{n-1}^{\prime} \bar{\epsilon}_{n} / \bar{\delta}_{n-1}^{\prime}, \\
\bar{\gamma}_{n}^{\prime} & =\bar{\gamma}_{n}-\bar{\beta}_{n-1}^{\prime} \bar{\epsilon}_{n} / \bar{\delta}_{n-1}^{\prime}, \quad \\
\bar{\delta}_{n}^{\prime} & =\bar{\delta}_{n}-\bar{\gamma}_{n-1}^{\prime} \bar{\epsilon}_{n} / \bar{\delta}_{n-1}^{\prime}, \quad \text { for } n \geq 3,
\end{aligned}
$$

and

$$
\begin{aligned}
\bar{\alpha}_{2}^{\prime \prime} & =\bar{\alpha}_{2}^{\prime}, \\
\bar{\beta}_{2}^{\prime \prime} & =\bar{\beta}_{2}^{\prime}, \\
\bar{\gamma}_{2}^{\prime \prime} & =\bar{\gamma}_{2}^{\prime}-\alpha_{1} \bar{\delta}_{2}^{\prime} / \gamma_{1}, \\
\bar{\alpha}_{n}^{\prime \prime} & =\bar{\alpha}_{n}^{\prime}, \\
\bar{\beta}_{n}^{\prime \prime} & =\bar{\beta}_{n}^{\prime}-\bar{\alpha}_{n-1}^{\prime \prime} \bar{\delta}_{n}^{\prime} / \bar{\gamma}_{n-1}^{\prime \prime}, \quad \\
\bar{\gamma}_{n}^{\prime \prime} & =\bar{\gamma}_{n}^{\prime}-\bar{\beta}_{n-1}^{\prime \prime} \bar{\delta}_{n}^{\prime} / \bar{\gamma}_{n-1}^{\prime \prime}, \quad \text { for } n \geq 2,
\end{aligned}
$$

give us a three-term recurrence relation,

$$
\bar{\alpha}_{n}^{\prime \prime} c_{n+1}+\bar{\beta}_{n}^{\prime \prime} c_{n}+\bar{\gamma}_{n}^{\prime \prime} c_{n-1}=0, \quad \text { for } n \geq 2 .
$$

\section{Appendix C}

Now we summarize here the relation between $V_{1}$ for multi-pole index $l$ and $V_{2}$ for multi-pole index $l+1$. If we introduce a new function $f$,

$$
f=\frac{r-1}{r^{2}},
$$

which vanishes at both boundaries, $r \rightarrow 1$ and $r \rightarrow \infty$, then two potential are given by

$$
\begin{aligned}
& V_{1}=+(l+1) \frac{d f}{d r_{*}}-4 f^{3}+(l+1)^{2} f^{2}, \\
& V_{2}=-(l+1) \frac{d f}{d r_{*}}-4 f^{3}+(l+1)^{2} f^{2} .
\end{aligned}
$$

If these two different potentials yield the same amplitudes of transmission and reflection, the following integrals for two potentials should be the same;

$$
I_{1}=\int V d r_{*},
$$




$$
\begin{aligned}
I_{2} & =\int V^{2} d r_{*}, \\
I_{3} & =\int\left(2 V^{3}+V^{\prime 2}\right) d r_{*}, \\
I_{4} & =\int\left(5 V^{4}+10 V V^{\prime 2}+V^{\prime \prime 2}\right) d r_{*}, \\
I_{5} & =\int\left(14 V^{5}+70 V^{2} V^{\prime 2}+14 V V^{\prime \prime 2}+V^{\prime \prime \prime 2}\right) d r_{*}, \\
I_{6} & =\ldots
\end{aligned}
$$

Due to the simple forms, Eq. (83) and Eq. (84), we can see the coincidence of these integrals, $I_{1} \sim I_{10}$, for two potentials. The explicit forms of above integrals, $I_{1} \sim I_{5}$, are given by

$$
\begin{aligned}
I_{1}= & \left(1+6 l+3 l^{2}\right) / 3 \\
I_{2}= & \left(4+18 l+93 l^{2}+84 l^{3}+21 l^{4}\right) / 630, \\
I_{3}= & \left(23+120 l+476 l^{2}+1560 l^{3}\right. \\
& \left.+1820 l^{4}+858 l^{5}+143 l^{6}\right) / 45045 \\
I_{4}= & \left(1648+8736 l+32716 l^{2}+105868 l^{3}\right. \\
& +252567 l^{4}+316540 l^{5}+203490 l^{6} \\
& \left.+64600 l^{7}+8075 l^{8}\right) / 9399380, \\
I_{5}= & \left(25160+125820 l+437634 l^{2}+1336860 l^{3}\right. \\
& +3430245 l^{4}+6563970 l^{5}+8099427 l^{6}+6085800 l^{7} \\
& \left.+2687895 l^{8}+642390 l^{9}+64239 l^{10}\right) / 1003917915 .
\end{aligned}
$$

\section{References}

[1] T. Regge and J. Wheeler, Phys. Rev. 108 (1957) 1063.

[2] F. Zerilli, Phys. Rev. D 2 (1971) 2141.

[3] F. Zerilli, Phys. Rev. D 9 (1974) 860.

[4] V. Moncrief, Phys. Rev. D 9 (1974) 2707; ibid. 10 (1974) 1057; ibid. 12 (1975) 1526.

[5] S. Teukolsky, Phys. Rev. Lett. 29 (1972) 1114.

[6] S. Chandrasekhar and S. Detweiler, Proc. Roy. Soc. London A344 (1975) 441.

[7] E. Leaver, Proc. Roy. Soc. London A402 (1985) 285.

[8] W. Gautschi, SIAM Rev 9 (1967) 24. 
[9] V. Ferrari and B. Mashhoon, Phys. Rev. D 30 (1984) 295.

[10] H. Blome and B. Mashhoon, Phys. Lett. 100A (1984) 231.

[11] B. Schutz and C. Will, Astrophys. J. 291 (1985) L33.

[12] S. Iyer and C. Will, Phys. Rev. D 35 (1987) 3621; S. Iyer, ibid. 35 (1987) 3632; E. Seidel and S. Iyer, ibid. 41 (1990) 374.

[13] E. Leaver, Phys. Rev. D 41 (1990) 2986.

[14] K. Kokkotas and B. Schutz, Phys. Rev. D 37 (1988) 3378.

[15] S. Chandrasekhar, The Mathematical Theory of Black Holes, (Clarendon, Oxford, 1983)

[16] S. Teukolsky and W. Press, Astrophys. J. 193 (1974) 443.

[17] S. Detweiler, Astrophys. J. 239 (1980) 292; Phys. Rev. Lett. 52 (1983) 67.

[18] M. Sasaki and T. Nakamura, Gen. Rel. Grav. 22 (1990) 1351. 


\begin{tabular}{|l|l|l|l|}
\hline & $s=2, l=2$ & $s=2, l=3$ & $s=2, l=4$ \\
\hline $\mathrm{n}=0$ & $(-0.083460,0.43134)$ & $(-0.085973,0.70430)$ & $(-0.087001,0.96576)$ \\
Leaver & $(-0.083645,0.43098)$ & & \\
WKB & $(-0.08349,0.43013)$ & $(-0.08596,0.70398)$ & $(-0.08700,0.96563)$ \\
$\mathrm{n}=1$ & $(-0.25498,0.40452)$ & $(-0.25992,0.68804)$ & $(-0.26212,0.95381)$ \\
Leaver & $(-0.257055,0.39309)$ & & \\
WKB & $(-0.25675,0.40076)$ & $(-0.26014,0.68701)$ & $(-0.26218,0.95339)$ \\
$\mathrm{n}=2$ & $(-0.44137,0.35340)$ & $(-0.44007,0.65624)$ & $(-0.44064,0.93020)$ \\
Leaver & $(-0.442035,0.353515)$ & & \\
WKB & $(-0.44210,0.35136)$ & $(-0.43986,0.65575)$ & $(-0.44056,0.93004)$ \\
\hline & $s=1, l=1$ & $s=1, l=2$ & $s=1, l=3$ \\
\hline $\mathrm{n}=0$ & $(-0.083460,0.43134)$ & $(-0.085973,0.70430)$ & $(-0.087001,0.96576)$ \\
Leaver & $(-0.08343,0.431415)$ & $(-0.086205,0.704075)$ & \\
WKB & $(-0.08349,0.43013)$ & $(-0.08596,0.70398)$ & $(-0.08700,0.96563)$ \\
$\mathrm{n}=1$ & $(-0.25498,0.40452)$ & $(-0.25992,0.68804)$ & $(-0.26212,0.95381)$ \\
Leaver & $(-0.259705,0.40602)$ & $(-0.26256,0.68315)$ & \\
WKB & $(-0.25675,0.40076)$ & $(-0.26014,0.68701)$ & $(-0.26218,0.95339)$ \\
$\mathrm{n}=2$ & $(-0.44137,0.35340)$ & $(-0.44007,0.65624)$ & $(-0.44064,0.93020)$ \\
Leaver & $(-0.44260,0.35347)$ & $(-0.4408,0.655675)$ & \\
WKB & $(-0.44210,0.35136)$ & $(-0.43986,0.65575)$ & $(-0.44056,0.93004)$ \\
\hline & $s=0, l=0$ & $s=0, l=1$ & $s=0, l=2$ \\
\hline $\mathrm{n}=0$ & $(-0.095844,0.13346)$ & $(-0.089384,0.37764)$ & $(-0.088748,0.62657)$ \\
WKB & $(-0.10371,0.12109)$ & $(-0.08936,0.37570)$ & $(-0.08873,0.62609)$ \\
$\mathrm{n}=1$ & $(-0.33065,0.092965)$ & $(-0.27614,0.34818)$ & $(-0.26909,0.60817)$ \\
WKB & $(-0.33742,0.09157)$ & $(-0.27828,0.34392)$ & $(-0.26944,0.60677)$ \\
$\mathrm{n}=2$ & $(-0.58833,0.075081)$ & $(-0.48643,0.29846)$ & $(-0.45820,0.57287)$ \\
WKB & $(-0.57164,0.05056)$ & $(-0.48145,0.29661)$ & $(-0.45750,0.57254)$ \\
\hline
\end{tabular}

Table 1: The computed quasinormal frequencies are listed. Our results are very consistent with the values of WKB quasinormal frequencies. The relative errors of WKB complex and real gravitational quasinormal frequencies for $l=2$ from our results are $(0.04 \%, 0.03 \%),(0.07 \%, 0.1 \%)$ and $(0.1 \%, 0.5 \%)$ for $n=0,1,2$, respectively. Leaver's value has rather large discrepancy of $3 \%$ for the real frequency of $n=1$ gravitational wave. 


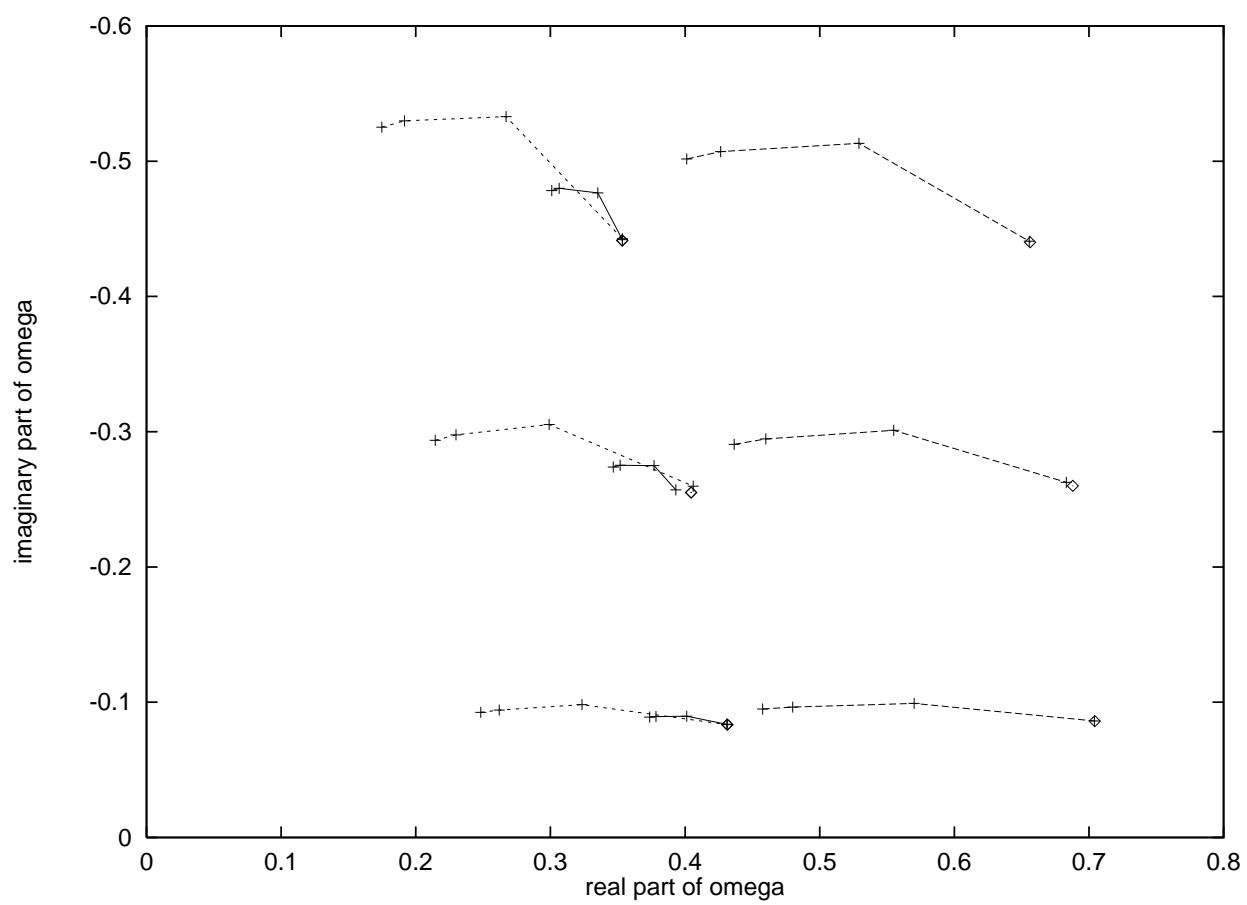

Figure 1: Quasinormal frequencies for $l=2$ gravitational wave and $l=1,2$ electromagnetic wave are plotted in the $\omega$-plane with the results of Leaver[13]. Each solid line is a trajectory of the first three lowest $l=2$ gravitational mode, parameterized by the charge $Q$. It has a tendency to coincide at the right end with $l=1$ electromagnetic mode which is shown as a small dashed line. Marks from left to right correspond to $Q=0,0.4,0.8,0.9999$ quasinormal frequencies of Leaver. The frequencies of extremal black holes we computed are plotted as diamonds. Dashed lines are $l=2$ electromagnetic modes. 


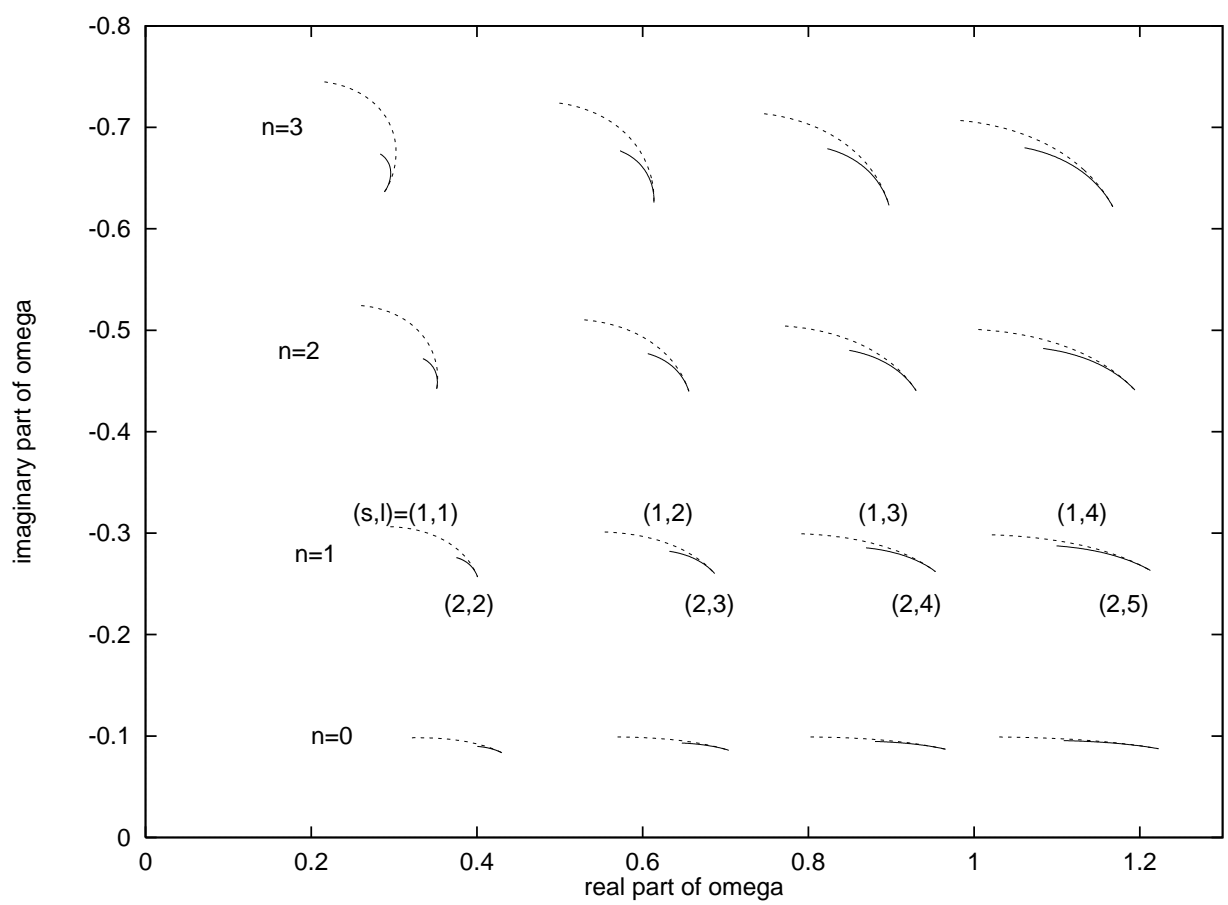

Figure 2: Solid lines and dashed lines are trajectories of the third order WKB quasinormal frequencies of the gravitational and electromagnetic wave, respectively. Each left endpoint of lines corresponds to the quasinormal frequency of a charged black hole of $Q=0.8$, and each right endpoint corresponds to the frequency in the limit of maximal charge. A trajectory of the gravitational quasinormal frequencies depicted in a solid line meets at the right end with a corresponding dashed line, which is a trajectory of the electromagnetic quasinormal frequencies belonging to lower multi-pole index by one. 